OPEN ACCESS

Edited by:

Fabio Grizzi

Humanitas Research Hospital, Italy

Reviewed by:

Felix K. H. Chun,

University Medical Center

Hamburg-Eppendorf, Germany

Naokazu lbuki,

Osaka Medical College, Japan

${ }^{*}$ Correspondence:

Ja Hyeon Ku

kuuro70@snu.ac.kr

Specialty section:

This article was submitted to

Genitourinary Oncology,

a section of the journal

Frontiers in Oncology

Received: 14 September 2018 Accepted: 06 December 2018

Published: 17 January 2019

Citation:

Yuk HD, Jeong CW, Kwak C, Kim HH and Ku JH (2019) Elevated Neutrophil

to Lymphocyte Ratio Predicts Poor

Prognosis in Non-muscle Invasive

Bladder Cancer Patients: Initial

Intravesical Bacillus Calmette-Guerin

Treatment After Transurethral

Resection of Bladder Tumor Setting.

Front. Oncol. 8:642.

doi: 10.3389/fonc.2018.00642

\section{Elevated Neutrophil to Lymphocyte Ratio Predicts Poor Prognosis in Non-muscle Invasive Bladder Cancer Patients: Initial Intravesical Bacillus Calmette-Guerin Treatment After Transurethral Resection of Bladder Tumor Setting}

\author{
Hyeong Dong Yuk ${ }^{1}$, Chang Wook Jeong ${ }^{2}$, Cheol Kwak ${ }^{2}$, Hyeon Hoe Kim ${ }^{2}$ and \\ Ja Hyeon $\mathrm{Ku}^{2 *}$
}

${ }^{1}$ Department of Urology, Inje University Sanggye Paik Hospital, Seoul, South Korea, ${ }^{2}$ Department of Urology, Seoul National University Hospital, Seoul, South Korea

The objective of this study was to investigate pretreatment systemic inflammatory response (SIR) markers in patients who underwent initial intravesical treatment for high-risk non-muscle invasive bladder cancer (NMIBC). A total of 385 patients who underwent initial intravesical Bacillus Calmette-Guerin treatment after transurethral resection of bladder tumor (TURB) were included. We analyzed the relationship between oncological outcomes and ratios of SIR markers, including neutrophil-to-lymphocyte ratio $(\mathrm{NLR})$, derived neutrophil-to-lymphocyte ratio (dNLR), and platelet-to-lymphocyte ratio (PLR). Each SIR marker was used for analysis. Their cut-off values were determined through receiver operation characteristics curves analysis. Patients were divided into two groups according to pretreatment NLR ( $<1.5$ vs. $\geq 1.5)$, dNLR ( $<1.2$ vs. $\geq 1.2)$, and PLR values $(171<$ vs. $\geq 171)$. Patients with NLR $\geq 1.5$ and $d N L R \geq 1.2$ were associated with poor prognosis in terms of overall survival and cause-specific survival. However, no serum SIR marker was associated with prognosis in recurrence-free survival or progression-free survival. Cox multivariate analysis revealed that age, NLR, dNLR, hemoglobin, and pathologic $T$ stage were significant factors predicting overall survival. Age, NLR, and pathologic T stage were significant factors predicting cancer-specific survival, NLR and tumor number were the most important predictors of bladder preserving survival. NLR before treatment was correlated with both oncological outcomes and survival outcome in NMIBC patients undergoing initial intravesical BCG treatment after TURB. Increased NLR reflects a poor prognosis of these outcomes.

Keywords: BCG, bladder cancer, neutrophil-to-lymphocyte ratio, systemic inflammatory response, non-muscle invasive bladder cancer 


\section{INTRODUCTION}

Bladder cancer is the 9th most commonly diagnosed cancer worldwide and the 13th most frequent cause of cancer death worldwide (1). It is also the 5th most common cancer in the United States. Bladder cancer is the most expensive cancer to treat (2).

Most bladder cancers are histologically diagnosed as urothelial cell carcinoma (UC). Ninety percent of UC cases originate in the bladder. Of these, $75 \%$ are diagnosed as non-muscle invasive bladder cancer (NMIBC) with mucosal or submucosal confined disease $(3,4)$. NMIBC has a wide range of tumor biology and heterogeneity (5). Because of this heterogeneity, there are various treatment options. It is difficult to determine standard treatment guidelines (5). Thus, several institutions have performed risk stratification based on the risk of recurrence and progression of tumors (5). Such stratification of risk groups based on recurrence and progression risk of tumors was a strategy used in an attempt to individualize and standardize treatment for specific patient populations (5). The European Association of Urology (EAU) and National Comprehensive Cancer Network (NCCN) guidelines are the two well-known treatment guidelines for this stratification $(3,6)$. The EAU guidelines defined NMIBC high risk group as T1 tumor, high grade tumor, carcinoma in situ (CIS), and Ta low grade tumor with multiple, recurrent, and large $(>3 \mathrm{~cm})$ tumor (3). The NCCN guidelines defined NMIBC high risk group as T1 tumor, high grade tumor, CIS (6). Patients whose NMIBC is high grade, T1, or carcinoma in situ (CIS) are at high risk of recurrence and progression (1). The standard treatment for NMIBC is transurethral resection of bladder tumor (TURB). However, the recurrence rate is as high as $80 \%$ within 5 years after initial TURB $(1,3)$. In addition, $30 \%$ of patients undergo radical cystectomy $(3,5)$. Most studies on SIR markers in bladder cancer have been focused on treatment for MIBC (7-13). These studies suggest that SIR markers may be useful for predicting tumor prognosis before treatment (713). Pretreatment patient-related factors may help predict the prognosis of cancer patients (14). In bladder cancer, factors associated with pretreatment may affect various treatments. They might affect treatment outcome of patients with NMIBC or MIBC. We hypothesized that pretreatment SIR markers in NMIBC patients could affect treatment outcome and prognosis of patients.

To prevent recurrence and rapid progression, we performed intravesical Bacillus Calmette-Guerin (BCG) therapy. In 1976, Morales et al. confirmed the efficacy of BCG therapy for bladder cancer patients and then for NMIBC patients $(3,6)$. Intravesical BCG treatment is now the standard treatment for high-risk NMIBC patients $(3,6,15)$. It is important to determine predictors for clinical outcome to improve BCG treatment decisions in these patients. Therefore, the objective of this study was to investigate associations between pretreatment systemic inflammatory response biomarkers and oncologic outcome of patients with UC.

\section{PATIENTS AND METHODS}

\section{Study Sample}

The Institutional Review Board of Seoul National University Hospital Clinical Research Institute approved this study (approval code: H-1710-033-891). This was a retrospective study. It was exempt from the requirement of patient written

TABLE 1 | Clinicopathological characteristics of NMIBC patients who underwent initial intravesical BCG treatment after TURB.

\begin{tabular}{|c|c|}
\hline Variables & Total $(N=385)$ \\
\hline Age & $72.6 \pm 10.6$ \\
\hline \multicolumn{2}{|l|}{ SEX } \\
\hline Man & 327 (84.9\%) \\
\hline Woman & $58(15.1 \%)$ \\
\hline $\mathrm{BMl}$ & $24.2 \pm 3.1$ \\
\hline Hypertension & $150(39.0 \%)$ \\
\hline Diabetes & 70 (18.2\%) \\
\hline Lung disease & $30(7.8 \%)$ \\
\hline White blood cell & $6.5 \pm 1.7$ \\
\hline Platelet & $218.9 \pm 56.7$ \\
\hline NLR & $2.2 \pm 1.2$ \\
\hline $\mathrm{dNLR}$ & $1.5 \pm 0.7$ \\
\hline PLR & $122.6 \pm 50.6$ \\
\hline Hemoglobin & $14.1 \pm 2.2$ \\
\hline Serum creatinine & $1.1 \pm 0.4$ \\
\hline eGFR & $73.0 \pm 17.5$ \\
\hline \multicolumn{2}{|l|}{ pT STAGE } \\
\hline Tis & 47 (12.2\%) \\
\hline $\mathrm{Ta}$ & $86(22.3 \%)$ \\
\hline $\mathrm{T} 1$ & 252 (65.5\%) \\
\hline \multicolumn{2}{|l|}{ TUMOR GRADE } \\
\hline None & $9(2.3 \%)$ \\
\hline Low grade & $50(13.0 \%)$ \\
\hline High grade & $326(84.7 \%)$ \\
\hline Concurrent CIS & 105 (27.3\%) \\
\hline LVI & $8(2.1 \%)$ \\
\hline \multicolumn{2}{|l|}{ TUMOR NUMBER } \\
\hline 1 & $148(38.4 \%)$ \\
\hline $2-7$ & $186(48.3 \%)$ \\
\hline$\geqq 3$ & $51(13.2 \%)$ \\
\hline \multicolumn{2}{|l|}{ TUMOR SIZE } \\
\hline$<3 \mathrm{~cm}$ & $290(75.3 \%)$ \\
\hline$\geqq 3 \mathrm{~cm}$ & 95 (24.7\%) \\
\hline Radical cystectomy (N/Person-Years) & $51(13.2 \%) / 84.8$ \\
\hline Upper urinary tract recurrence (N/Person-Years) & $16(4.2 \%) / 72.1$ \\
\hline Recurrence (N/Person-Years) & $186(48.3 \%) / 669.3$ \\
\hline Progression (N/Person-Years) & $44(11.4 \%) / 116.1$ \\
\hline Cancer specific mortality (N/Person-Years) & $18(4.7 \%) / 103.6$ \\
\hline Total mortality (N/Person-Years) & $70(18.2 \%) / 300.6$ \\
\hline
\end{tabular}

NMIBC, non-muscle invasive bladder cancer; BMI, body mass index; SIR, systemic inflammatory response; NLR, neutrophil-to-lymphocyte ratio; dNLR, derived NLR; PLR, platelet-lymphocyte ratio; CIS, carcinoma in situ. 

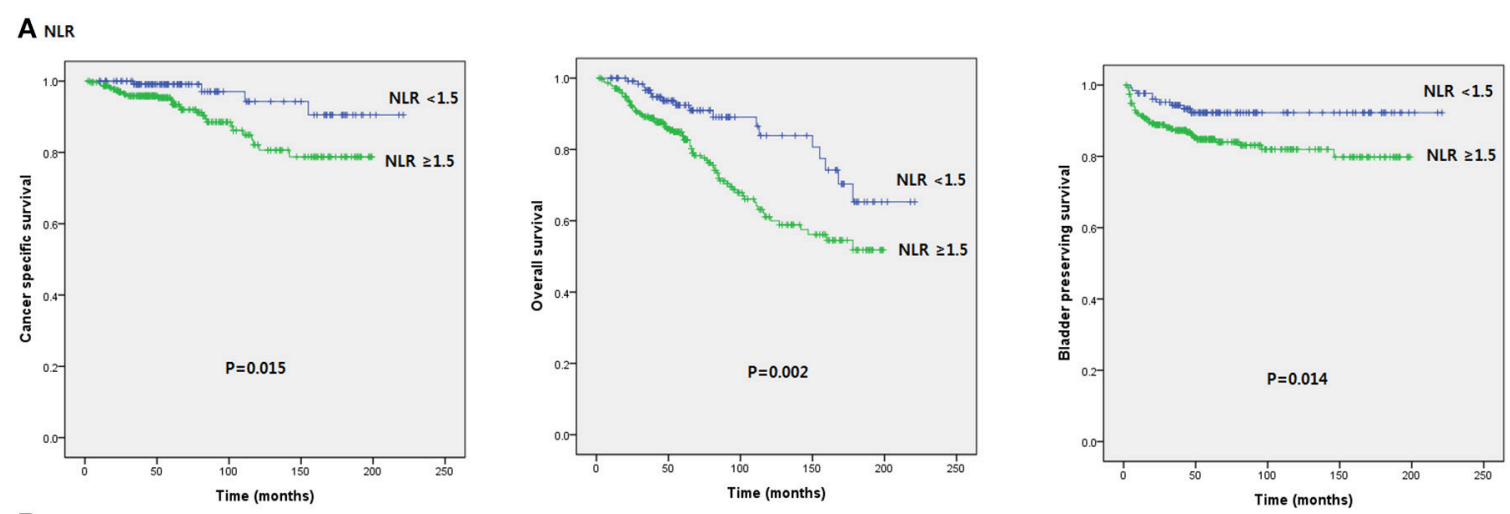

B dNLR
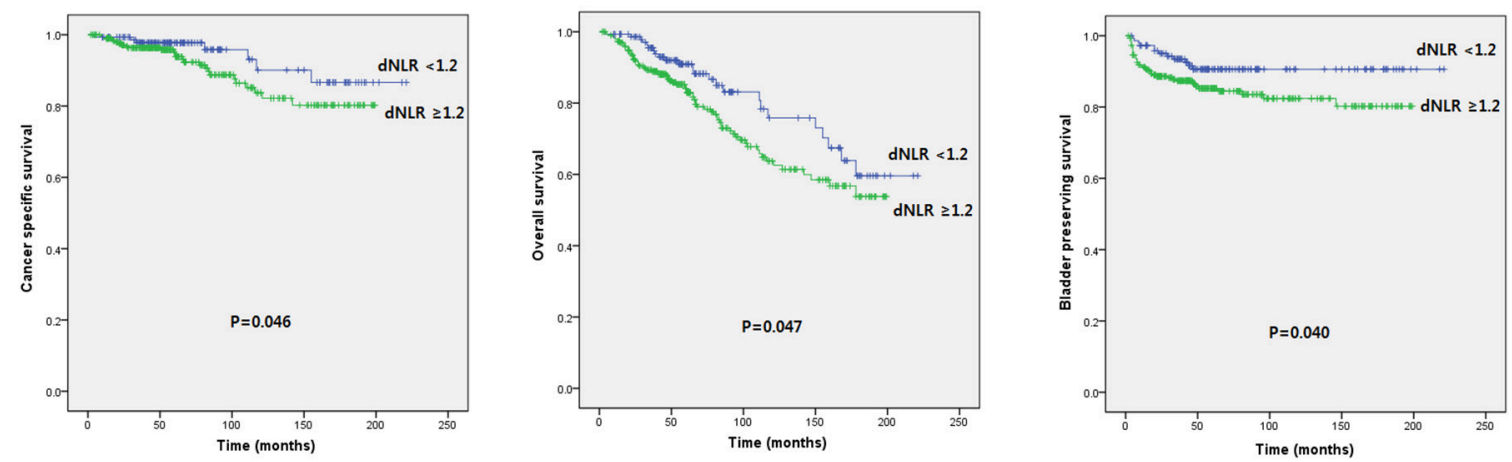

$C_{\text {PLR }}$
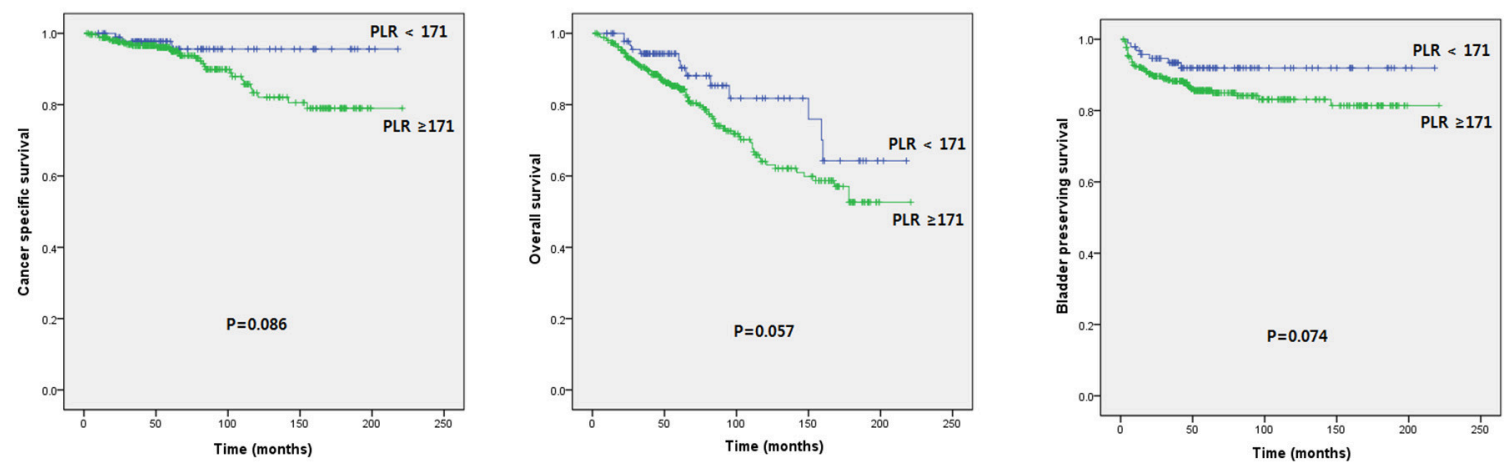

FIGURE 1 | Kaplan-Meier survival curves of oncological outcomes according to pretreatment SIR markers in initial BCG patients after TURB.

consent. The study protocol and all related items followed the Declaration of Helsinki guidelines. We retrospectively reviewed medical records of 3,127 patients who received TURB at Seoul National University Hospital from 1991 to 2015. Patients who underwent initial intravesical BCG treatment after TURB were included. There was a difference in treatment duration from 1991 to 2015. Indication and recommended method of intravesical BCG treatment also differed for different ages. Therefore, we retrospectively included patients who underwent intravesical BCG treatment among patients who met our defined high-risk group criteria. All patients were high-risk NMIBC patients with T1, high-grade tumor, or CIS. Patients without UC, with short follow-up ( $<2$ years), and those who were suspected of infections accompanied by fever or leukocytosis were excluded. Finally, 385 patients were included.

\section{Study Design}

Pretreatment systemic inflammatory response markers were investigated. We collected clinicopathological information from patient's medical records at the time of initial intravesical treatment after TURB. Clinicopathological information included age, sex, body mass index (BMI), hypertension, diabetes, lung disease, absolute neutrophil, lymphocyte, and platelet counts, hemoglobin, pre-intravesical treatment NLR, dNLR, and PLR values, creatinine, estimated glomerular filtration ratio (eGFR), pathological $\mathrm{T}$ stage, tumor grade, CIS, lymphovascular invasion (LVI), tumor number, and 
TABLE 2 | Multivariate Cox proportional hazard ratio analysis to identify predictive factors for overall survival.

\begin{tabular}{|c|c|c|c|c|}
\hline \multirow[t]{2}{*}{ Parameter } & \multicolumn{2}{|c|}{ Univariate } & \multicolumn{2}{|c|}{ Multivariate } \\
\hline & HR (95\% Cl) & $P$-value & HR (95\% Cl) & $P$-value \\
\hline Age & $1.11(1.07-1.15)$ & 0.000 & $1.08(1.05-1.10)$ & 0.000 \\
\hline \multicolumn{5}{|l|}{ NLR } \\
\hline$N L R<1.5$ & Reference & & Reference & \\
\hline$N L R \geq 1.5$ & $1.94(0.82-4.58)$ & 0.012 & $2.24(1.26-3.96)$ & 0.005 \\
\hline \multicolumn{5}{|l|}{ dNLR } \\
\hline $\mathrm{dNLR}<1.2$ & Reference & & Reference & \\
\hline $\mathrm{dNLR} \geq 1.2$ & $2.12(1.16-4.11)$ & 0.010 & $1.65(0.98-2.55)$ & 0.050 \\
\hline \multicolumn{5}{|l|}{ PLR } \\
\hline PLR $<171$ & Reference & & Reference & \\
\hline PLR > 171 & $0.92(0.46-1.82)$ & 0.819 & $0.74(0.39-1.39)$ & 0.357 \\
\hline $\mathrm{Hb}$ & $0.82(0.72-0.94)$ & 0.005 & $0.80(0.68-0.95)$ & 0.011 \\
\hline \multicolumn{5}{|l|}{ T STAGE } \\
\hline$<\mathrm{T} 1$ & Reference & & Reference & \\
\hline $\mathrm{T} 1$ & 1.98 (1.19-3.30) & 0.008 & 1.99 (1.09-3.64) & 0.025 \\
\hline \multicolumn{5}{|l|}{ TUMOR GRADE } \\
\hline Low & Reference & & Reference & \\
\hline High & $2.15(0.88-5.24)$ & 0.089 & $1.76(0.73-4.25)$ & 0.209 \\
\hline Concomitant CIS & 1.65 (0.93-2.93) & 0.089 & $1.95(0.98-3.86)$ & 0.054 \\
\hline LVI & $4.71(1.15-19.32)$ & 0.031 & $1.67(0.59-4.73)$ & 0.335 \\
\hline Tumor Number & & 0.264 & & 0.780 \\
\hline 1 & Reference & & Reference & \\
\hline $2 \sim 7$ & 1.57 (0.89-2.78) & 0.123 & $1.21(0.70-2.04)$ & 0.482 \\
\hline$\geq 3$ & $1.07(0.44-2.57)$ & 0.888 & $1.11(0.48-2.52)$ & 0.809 \\
\hline \multicolumn{5}{|l|}{ TUMOR SIZE } \\
\hline$<3 \mathrm{~cm}$ & Reference & & Reference & \\
\hline$\geq 3 \mathrm{~cm}$ & $1.30(0.76-2.24)$ & 0.332 & $1.61(0.83-3.13)$ & 0.162 \\
\hline
\end{tabular}

NLR, neutrophil-to-lymphocyte ratio; $d N L R$, derived NLR; PLR, platelet-lymphocyte ratio; $H b$, hemoglobin; CIS, carcinoma in situ; LVI, lymphovascular invasion.

tumor size. We also collected various oncologic prognoses and outcomes, including upper urinary tract recurrence, recurrence in bladder, history and timing of radical cystectomy, progression, cancer-specific mortality, and overall mortality. SIR markers calculated included neutrophil-to-lymphocyte ratio (NLR, absolute neutrophil count/lymphocyte count), derived neutrophil-to-lymphocyte ratio [dNLR (absolute neutrophil count/white blood cell count) - neutrophil count], and plateletto-lymphocyte ratio (PLR, absolute platelet count/lymphocyte count).

We performed TURB and intravesical treatment according to established principles of our hospital. Specimens resected by TURB were histologically examined and reviewed by two experienced urological pathologists. They determined the TNM stage and tumor grade according to the 2010 American Joint Committee on Cancer classification and the 2004 World Health Organization/International Society of Urologic Pathology consensus classifications principles. Almost all patients were followed-up every 3 months for 3 years first. They were then followed up every 6 months for 2 more years (year 5) and annually thereafter. At each
TABLE 3 | Multivariate Cox proportional hazard ratio analysis to identify predictive factors for cancer specific survival.

\begin{tabular}{|c|c|c|c|c|}
\hline \multirow[t]{2}{*}{ Parameter } & \multicolumn{2}{|c|}{ Univariate } & \multicolumn{2}{|c|}{ Multivariate } \\
\hline & HR $(95 \% \mathrm{Cl})$ & $P$-value & HR (95\% Cl) & $P$-value \\
\hline Age & $1.18(1.12-1.25)$ & 0.000 & $1.10(1.03-1.16)$ & 0.003 \\
\hline \multicolumn{5}{|l|}{ NLR } \\
\hline$N L R<1.5$ & Reference & & Reference & \\
\hline$N L R \geq 1.5$ & $3.17(1.22-10.85)$ & 0.003 & 2.03 (1.03-3.99) & 0.039 \\
\hline \multicolumn{5}{|l|}{ dNLR } \\
\hline $\mathrm{dNLR}<1.2$ & Reference & & Reference & \\
\hline $\mathrm{dNLR} \geq 1.2$ & $1.95(0.61-6.16)$ & 0.194 & 1.25 (0.38-4.13) & 0.709 \\
\hline \multicolumn{5}{|l|}{ PLR } \\
\hline PLR $<171$ & Reference & & Reference & \\
\hline PLR > 171 & $2.19(0.49-9.75)$ & 0.302 & $1.52(0.29-7.83)$ & 0.615 \\
\hline $\mathrm{Hb}$ & $0.94(0.70 .-1.24)$ & 0.939 & $0.90(0.65-1.26)$ & 0.555 \\
\hline \multicolumn{5}{|l|}{ T STAGE } \\
\hline$<\mathrm{T} 1$ & Reference & & Reference & \\
\hline $\mathrm{T} 1$ & $2.77(1.42-5.40)$ & 0.003 & $2.36(1.52-3.66)$ & 0.005 \\
\hline \multicolumn{5}{|l|}{ TUMOR GRADE } \\
\hline Low & Reference & & Reference & \\
\hline High & $1.11(0.31-3.96)$ & 0.871 & $1.45(0.36-5.83)$ & 0.602 \\
\hline Concomitant CIS & $6.72(0.88-51.16)$ & 0.066 & $2.43(0.28-21.23)$ & 0.422 \\
\hline LVI & $3.02(0.35-25.99)$ & 3.025 & $1.25(0.16-9.75)$ & 0.832 \\
\hline Tumor Number & & 0.125 & & 0.543 \\
\hline 1 & Reference & & Reference & \\
\hline $2 \sim 7$ & 3.63 (1.01-12.99) & 0.047 & 2.05 (0.56-7.52) & 0.280 \\
\hline$\geq 3$ & $1.97(0.32-12.15)$ & 0.464 & $1.50(0.22-10.11)$ & 0.675 \\
\hline \multicolumn{5}{|l|}{ TUMOR SIZE } \\
\hline$<3 \mathrm{~cm}$ & Reference & & Reference & \\
\hline$\geq 3 \mathrm{~cm}$ & $5.85(0.77-44.58)$ & 0.088 & 4.99 (0.63-39.7) & 0.129 \\
\hline
\end{tabular}

NLR, neutrophil-to-lymphocyte ratio; $d N L R$, derived NLR; PLR, platelet-lymphocyte ratio; $H b$, hemoglobin; CIS, carcinoma in situ; LVI, lymphovascular invasion.

follow-up, basic laboratory tests, urine analysis, urine cytology, and cystoscopy were performed. Computed tomography (CT) was performed annually to check progression and upper tract recurrence. Intravesical BCG treatment was performed. The BCG schedule was as follows: BCG induction therapy (weekly BCG instillation for the first 6 weeks) and maintenance therapy for up to 3 years (3 weekly maintenance instillation for $3,6,12,18,24,30$, and 36 months).

\section{Statistical Analyses}

The primary end point was overall survival (OS). Secondary end points were recurrence-free survival (RFS), progressionfree survival (PFS), bladder preserving survival (BPS), and cancer-specific survival (CSS). Kaplan-Meier survival analysis and log rank test were used for all survival analyses. Cox proportional hazard regression analysis was used to analyze various independent predictors of oncological outcomes. Univariate analysis was used for various factors related to patient characteristics and tumor characteristics before initial intravesical BCG treatment. Multivariate analysis was performed 
TABLE 4 | Multivariate Cox proportional hazard ratio analysis to identify predictive factors for Bladder preserving survival.

\begin{tabular}{|c|c|c|c|c|}
\hline \multirow[t]{2}{*}{ Parameter } & \multicolumn{2}{|c|}{ Univariate } & \multicolumn{2}{|c|}{ Multivariate } \\
\hline & HR(95\% Cl) & $P$-value & $\mathrm{HR}(95 \% \mathrm{Cl})$ & $P$-value \\
\hline Age & $1.01(0.98-1.05)$ & 0.261 & $0.99(0.97-1.02)$ & 0.691 \\
\hline \multicolumn{5}{|l|}{ NLR } \\
\hline NLR $<1.5$ & Reference & & Reference & \\
\hline$N L R \geq 1.5$ & $3.25(1.34-7.86)$ & 0.009 & $2.97(1.19-7.42)$ & 0.019 \\
\hline \multicolumn{5}{|l|}{ dNLR } \\
\hline $\mathrm{dNLR}<1.2$ & Reference & & Reference & \\
\hline $\mathrm{dNLR} \geq 1.2$ & $1.74(0.81-3.74)$ & 0.154 & $1.32(0.64-2.69)$ & 0.444 \\
\hline \multicolumn{5}{|l|}{ PLR } \\
\hline PLR $<171$ & Reference & & Reference & \\
\hline PLR > 171 & $2.17(0.89-5.29)$ & 0.088 & $1.43(0.58-3.55)$ & 0.439 \\
\hline $\mathrm{Hb}$ & $0.96(0.81-1.13)$ & 0.641 & $0.91(0.76-1.10)$ & 0.342 \\
\hline \multicolumn{5}{|l|}{ T STAGE } \\
\hline$<\mathrm{T} 1$ & Reference & & Reference & \\
\hline $\mathrm{T} 1$ & $1.31(0.69-2.49)$ & 0.409 & $1.23(0.64-2.37)$ & 0.528 \\
\hline \multicolumn{5}{|l|}{ TUMOR GRADE } \\
\hline Low & Reference & & Reference & \\
\hline High & $2.31(0.80-6.69)$ & 0.121 & $1.42(0.63-3.19)$ & 0.389 \\
\hline Concomitant CIS & $1.25(0.63-2.50)$ & 0.520 & $1.31(0.65-2.67)$ & 0.446 \\
\hline LVI & $1.07(0.12-8.88)$ & 0.950 & 1.69 (0.39-7.26) & 0.479 \\
\hline Tumor Number & & 0.48 & & 0.041 \\
\hline 1 & Reference & & Reference & \\
\hline $2 \sim 7$ & $2.18(1.07-4.42)$ & 0.031 & $2.10(1.04-4.24)$ & 0.038 \\
\hline$\geq 3$ & $2.43(0.96-6.16)$ & 0.042 & $2.84(1.18-6.82)$ & 0.019 \\
\hline \multicolumn{5}{|l|}{ TUMOR SIZE } \\
\hline$<3 \mathrm{~cm}$ & Reference & & Reference & \\
\hline$\geq 3 \mathrm{~cm}$ & $1.89(0.86-4.18)$ & 0.115 & $2.11(0.95-4.67)$ & 0.066 \\
\hline
\end{tabular}

$N L R$, neutrophil-to-lymphocyte ratio; $d N L R$, derived NLR; PLR, platelet-lymphocyte ratio; $H b$, hemoglobin; CIS, carcinoma in situ; LVI, lymphovascular invasion.

only for variables with $P<0.1$ in univariate analysis. By identifying explanatory variables that were significantly related to response variable, dozens of variables were considered one at a time. A less restrictive level $(P<0.1)$ is often used to identify many explanatory variables that can be associated with response variables in univariate analysis. Thus, we used a limited number of variables with $P<0.1$ in the univariate analysis for the multiple regression model. We used receiver-operating characteristic (ROC) curve to find appropriate cut off values for SIR markers. The optimal cut-off value was selected as the point at which the sensitivity and specificity were maximized for the prediction of oncologic outcomes. In the ROC curve analysis (AUC $=0.623$; 95\% CI $=0.55-0.69 ; p<0.001)$, dNLR $(\mathrm{AUC}=0.592 ; 95 \% \mathrm{CI}=0.52-0.67 ; p<0.001)$, and PLR (AUC $=0.674 ; 95 \% \mathrm{CI}=0.50-0.64 ; p<0.001$ ) showed predictive power to distinguish OS. Continuous variables are presented as mean values with standard deviation (SD) while categorical variables are presented as ratio of events (\%). Statistical significance was defined at $p \leq 0.05$. IBM SPSS Statistics version 22.0 (IBM, Armonk, NY, USA) was used for all statistical analysis.

\section{RESULTS}

\section{Clinicopathological Characteristics of Patients Who Underwent Initial Intravesical BCG Treatment After TURB}

A total of 385 high risk NMIBC cases were diagnosed after TURB and initial intravesical BCG treatment was performed. The mean follow-up time was 80 months [Standard deviation \pm 50.7 months]. Most (84.9\%) patients were males. Nearly half (48.3\%) of these patients experienced recurrence and $11.4 \%$ of patients had disease progression. Radical cystectomy was performed for $13.2 \%$ of patients. The overall mortality rate was $18.2 \%$. Cancerspecific mortality rate was $4.7 \%$. The mean time to recurrence was 43 months while the mean time to progression was 31 months. Median values of SIR markers were: NLR, 1.86 (IQR: 0.26-3.50); dNLR, 1.36 (IQR: 0.22-3.09); and PLR, 113.25: (IQR 35.3-200.8) (Table 1).

\section{Correlation of Serum SIR Markers With Oncologic and Survival Outcomes}

Patients were divided into two groups according to pretreatment NLR ( $<1.5$ vs. $\geq 1.5$ ), dNLR ( $<1.2$ vs. $\geq 1.2)$, and PLR values $(171<$ vs. $\geq 171)$. Patients with NLR $\geq 1.5$ and $d N L R \geq 1.2$ were associated with poor prognosis in terms of OS ( $p=0.002$ and $p=$ 0.047 , respectively), CSS ( $p=0.015$ and $p=0.046$, respectively), and BPS ( $p=0.014$ and $p=0.040$, respectively). However, no serum SIR marker was associated with prognosis in terms of RFS or PFS. Unexpectedly, PLR did not help predict any oncologic or survival outcome in patients with intravesical BCG treatment (Figure 1).

\section{Important Predictors of Oncological Outcomes by Cox Multivariate Regression Analysis}

Important predictors of OS in multivariate analysis were age, NLR, dNLR, $\mathrm{Hb}$, and pathologic $\mathrm{T}$ stage (Table 2). Important predictors of CSS were age, NLR, and pathologic T stage (Table 3). NLR and tumor number were the most important predictors for BPS (Table 4). ROC curve analysis showed that NLR and dNLR had predictive power for OS of patients with TURB after initial intravesical BCG treatment (area under the curve $[\mathrm{AUC}]=0.59 ; 95 \% \mathrm{CI}=0.52-0.65 ; p<0.001$ and $\mathrm{AUC}$ $=0.55 ; 95 \% \mathrm{CI}=0.49-0.62 ; p<0.001)$.

\section{Subgroup Analysis For Patients With Carcinoma in situ}

In subgroup analysis of CIS patients, patients with NLR $\geq 1.5$ and $\mathrm{dNLR} \geq 1.2$ had poor prognosis for OS ( $p=0.04$ and $p=0.034$, respectively) and CSS ( $p=0.001$ and $p=0.014$, respectively). However, there was no statistically significant relationship of NLR or dNLR with BPS, PFS, or RFS. PLR was not significantly associated with oncologic survival outcomes (Figure 2). 

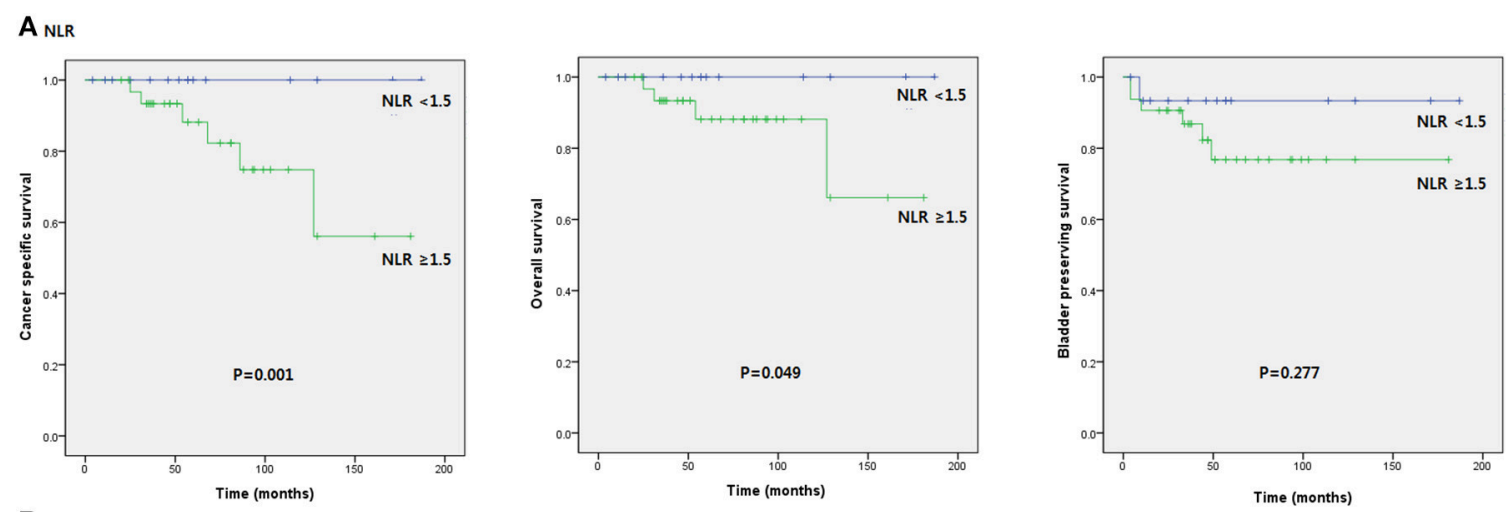

B dNLR
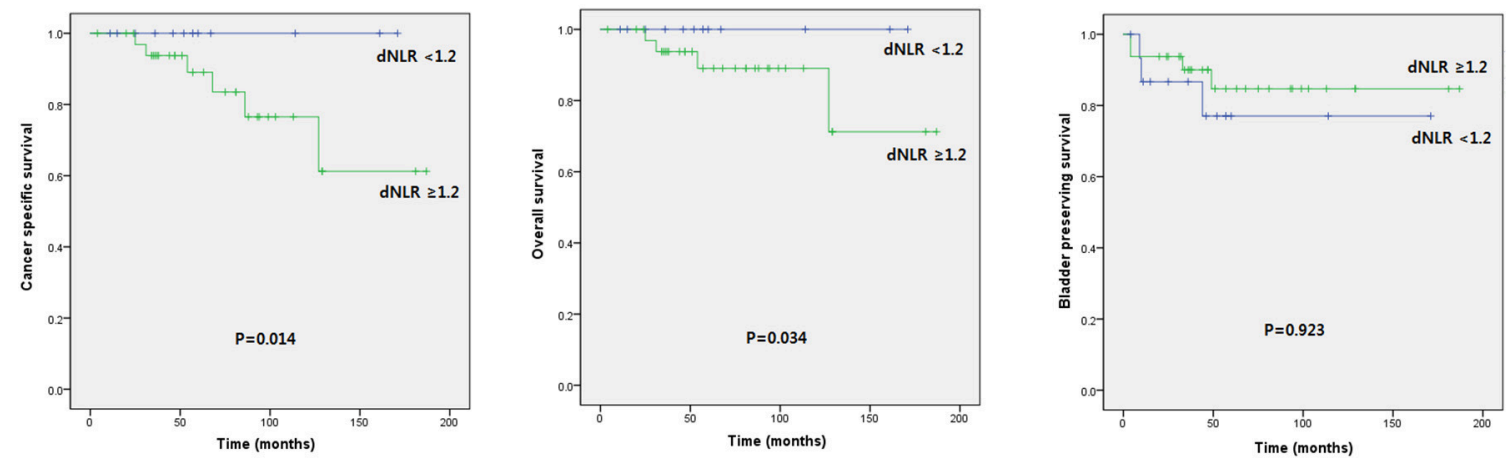

$C_{\text {PLR }}$
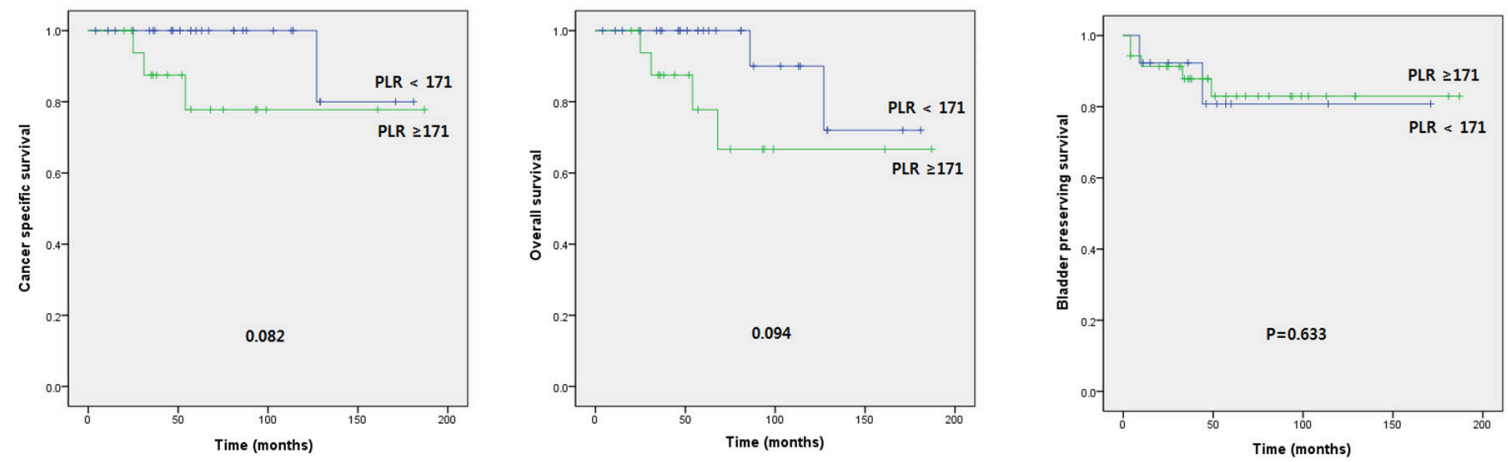

FIGURE 2 | Kaplan-Meier survival curves of oncological outcomes according to pretreatment SIR markers in subgroup of patients with CIS.

\section{DISCUSSION}

Cancer is affected by a variety of factors, including tumorrelated and patient-related factors. Until recently, tumorrelated factors, such as pathologic stage and grade have been used predominantly in predicting prognosis in cancer patients. However, patient-related factors can also predict the prognosis of cancer patients. These include weight loss, performance status, and SIR. Since Virchow first observed the presence of leukocytes in neoplastic tissue, the association of SIR with tumor progression has been demonstrated (14). Tumors can produce an intrinsic inflammatory response and a protumorigenic microenvironment. Increased inflammation in such a microenvironment promotes cancer activity (16).
Accelerated cancer activity will act on immune cells, leading to an increase in systemic immune responses (16). Many recent clinical studies support that SIR is a significant predictor of prognosis in various types of cancer, including esophagus, gastric, colorectal, liver, pancreas, breast, ovaries, cervix, prostate, kidney, and bladder cancers (7-13).

There are few studies on the association between SIR markers and NMIBC. Most studies have been performed on muscle invasive bladder cancer (MIBC) and radical cystectomy. These studies suggest that NLR before radical cystectomy may help predict tumor prognosis (17-21). The cut-off value of preoperative NLR ranges from 2 to 3 . Increased NLR is an independent predictor of OS, CSS, and RFS (17-21). A recent meta-analysis using 23 studies and $\sim 6,000$ MIBC patients has 
shown that increased NLR is highly relevant to OS, CSS, and RFS (22). In our study, increased NLR was a predictor of OS and CSS. In addition, increased NLR was helpful in predicting BPS. However, unlike previous studies, there was no significant correlation between increased NLR and RFS predictions (Figure 1). In multivariate analysis, age, increased NLR, and pathologic $\mathrm{T}$ stage were important predictors of OS and CSS while increased NLR and tumor numbers were important predictors of BPS (Tables 1-3). Cut off values of NLR and dNLR from ROC curve analysis were 1.5 and 1.2, respectively, which were slightly lower than those (between 2 and 3 ) reported in previous studies (17-21). Such discrepancy might be due to different timing of execution, difference in disease status, differing immune responses of the host, and differences in tumor characteristics. The difference between the current study and prior studies was that NMIBC patients were our study subject and the analysis was concerned about SIR marker before initial BCG treatment in high-risk pathologic patients after TURB, not surgery. Previous studies have shown that increased NLR before TURB in NMIBC patients is a predictor of OS and CSS (23). The cut-off value was 2.0 for NLR and 1.5 for dNLR (23). There may be difference between surgery and intravesical BCG. However, both NMIBC studies showed similar results (23). NLR was associated with survival outcome. It was not significantly associated with oncological outcomes, such as recurrence or progression (23). In another study, preoperative NLR was favorable to predict recurrence and progression in MIBC patients with initial TURB (24). This is different from results of the present study. A subgroup analysis of the adjuvant BCG group in a recently published NMIBC study has shown that high NLR is associated with disease progression, but not disease recurrence (25).

Unlike previous studies, our results showed that pretreatment NLR or dNLR did not increase the presence of tumor grade, number, size, concomitant CIS, or LVI. This may be due to insufficient patient pool. In addition, our study was performed

\section{REFERENCES}

1. Babjuk M, Bohle A, Burger M, Capoun O, Cohen D, Comperat EM, et al. EAU guidelines on non-muscle-invasive urothelial carcinoma of the bladder: update 2016. Eur Urol. (2017) 71:447-61. doi: 10.1016/j.eururo.2016. 05.041

2. Balkwill F, Mantovani A. Inflammation and cancer: back to Virchow? Lancet (2001) 357:539-45. doi: 10.1016/S0140-6736(00) 04046-0

3. Burger M, Catto JW, Dalbagni G, Grossman HB, Herr H, Karakiewicz P, et al. Epidemiology and risk factors of urothelial bladder cancer. Eur Urol. (2013) 63:234-41. doi: 10.1016/j.eururo.2012.07.033

4. Crumley AB, McMillan DC, McKernan M, Going JJ, Shearer CJ, Stuart RC. An elevated C-reactive protein concentration, prior to surgery, predicts poor cancer-specific survival in patients undergoing resection for gastro-oesophageal cancer. Br J Cancer (2006) 94:1568-71. doi: $10.1038 /$ sj.bjc.6603150

5. Demirtas A, Sabur V, Akinsal EC, Demirci D, Ekmekcioglu O, Gulmez I, et al. Can neutrophil-lymphocyte ratio and lymph node density be used as prognostic factors in patients undergoing radical cystectomy? Sci World J. (2013) 2013:703579. doi: 10.1155/2013/703579 on patients after TURB. In mechanistic view, advanced stage and aggressiveness may be associated with increased host immune response. However, in this study, all patients were NMIBC patients with low tumor burden after TURB. Such patient status seems to be difficult to reflect characteristics of the tumor.

This study has several limitations. First, it was a retrospective study of a single institution. Thus, it cannot be free from selection bias. Second, the timing of pretreatment NLR measurements was not exactly the same. Third, a single test does not represent the entire systemic immune response state. Fourth, values and confidence intervals of the AUC of the ROC curves cannot show solid results to establish these values as cutoff points with sufficient prediction.

In conclusion, NLR before treatment was correlated with both oncological outcomes and survival outcome in NMIBC patients undergoing initial intravesical BCG treatment after TURB. Increased NLR reflects poor prognosis of these outcomes. Although large-scale prospective studies are needed to apply NLR to clinical practice, NLR will be a useful tool to help improve risk assessment and treatment guidelines for intravesical treatment in high-risk NMIBC patients.

\section{AUTHOR CONTRIBUTIONS}

$\mathrm{HY}, \mathrm{CJ}, \mathrm{HK}$, and JK contributed to the concept and project planning. HY, CJ, and CK contributed significantly to the writing of this paper. JK contributed significantly to the editing of the final draft.

\section{FUNDING}

This study was supported by the National Research Foundation of Korea (NRF) grant funded by the Korea government (MSIP) (Grant number: 2016R1A2B4011623) to JK and (Grant number: 2015R1A2A2A01006813) to CK.

6. Dutta S, Crumley AB, Fullarton GM, Horgan PG, McMillan DC. Comparison of the prognostic value of tumour and patient related factors in patients undergoing potentially curative resection of gastric cancer. Am J Surg. (2012) 204:294-9. doi: 10.1016/j.amjsurg.2011.10.015

7. Grivennikov SI, Greten FR, Karin M. Immunity, inflammation, and cancer. Cell (2010) 140:883-99. doi: 10.1016/j.cell.2010.01.025

8. Hall MC, Chang SS, Dalbagni G, Pruthi RS, Seigne JD, Skinner EC, et al. Guideline for the management of nonmuscle invasive bladder cancer (stages Ta, T1, and Tis): 2007 update. J Urol. (2007) 178:2314-30. doi: 10.1016/j.juro.2007.09.003

9. Hashimoto K, Ikeda Y, Korenaga D, Tanoue K, Hamatake M, Kawasaki $\mathrm{K}$, et al. The impact of preoperative serum C-reactive protein on the prognosis of patients with hepatocellular carcinoma. Cancer (2005) 103:185664. doi: $10.1002 / \mathrm{cncr} .20976$

10. Hermanns T, Bhindi B, Wei Y, Yu J, Noon AP, Richard PO, et al. Pre-treatment neutrophil-to-lymphocyte ratio as predictor of adverse outcomes in patients undergoing radical cystectomy for urothelial carcinoma of the bladder. $\mathrm{Br} \mathrm{J}$ Cancer (2014) 111:444-51. doi: 10.1038/bjc.2014.305

11. Heys SD, Walker LG, Deehan DJ, Eremin OE. Serum albumin: a prognostic indicator in patients with colorectal cancer. J R Coll Surg Edinb. (1998) 43:163-8. 
12. Kamat AM, Witjes JA, Brausi M, Soloway M, Lamm D, Persad R, et al. Defining and treating the spectrum of intermediate risk nonmuscle invasive bladder cancer. J Urol. (2014) 192:305-15. doi: 10.1016/j.juro.2014.02.2573

13. Kang M, Jeong CW, Kwak C, Kim HH, Ku JH. Preoperative neutrophil-lymphocyte ratio can significantly predict mortality outcomes in patients with non-muscle invasive bladder cancer undergoing transurethral resection of bladder tumor. Oncotarget (2017) 8:12891-901. doi: 10.18632/oncotarget.14179

14. Ferlay J, Soerjomataram I, Dikshit R, Eser S, Mathers C, Rebelo M, et al. Cancer incidence and mortality worldwide: sources, methods and major patterns in GLOBOCAN 2012. Int J Cancer (2015) 136:E359-386. doi: 10.1002/ijc.29210

15. Espinosa E, Feliu J, Zamora P, Gonzalez Baron M, Sanchez JJ, Ordonez A, et al. Serum albumin and other prognostic factors related to response and survival in patients with advanced non-small cell lung cancer. Lung Cancer (1995) 12:67-76. doi: 10.1016/0169-5002(95)00407-R

16. Gondo T, Nakashima J, Ohno Y, Choichiro O, Horiguchi Y, Namiki $\mathrm{K}$, et al. Prognostic value of neutrophil-to-lymphocyte ratio and establishment of novel preoperative risk stratification model in bladder cancer patients treated with radical cystectomy. Urology (2012) 79:1085-91. doi: 10.1016/j.urology.2011.11.070

17. Kim HS, Ku JH. Systemic inflammatory response based on neutrophil-tolymphocyte ratio as a prognostic marker in bladder cancer. Dis Markers (2016) 2016:8345286. doi: 10.1155/2016/8345286

18. Lis CG, Grutsch JF, Vashi PG, Lammersfeld CA. Is serum albumin an independent predictor of survival in patients with breast cancer? JPEN J Parenter Enteral Nutr. (2003) 27:10-5. doi: 10.1177/014860710302700110

19. Mano R, Baniel J, Shoshany O, Margel D, Bar-On T, Nativ O, et al. Neutrophil-to-lymphocyte ratio predicts progression and recurrence of non-muscle-invasive bladder cancer. Urol. Oncol. (2015) 33:67 e61-67. doi: 10.1016/j.urolonc.2014.06.010

20. Marchioni M, Primiceri G, Ingrosso M, Filograna R, Castellan P, De Francesco $\mathrm{P}$, et al. The clinical use of the neutrophil to lymphocyte ratio (nlr) in urothelial cancer: a systematic review. Clin Genitourin Cancer (2016) 14:47384. doi: 10.1016/j.clgc.2016.04.008

21. Mbeutcha A, Shariat SF, Rieken M, Rink M, Xylinas E, Seitz C, et al. Prognostic significance of markers of systemic inflammatory response in patients with non-muscle-invasive bladder cancer. Urol. Oncol. (2016) 34:483 e417-24. doi: 10.1016/j.urolonc.2016.05.013

22. Morales A, Eidinger D, Bruce AW. Intracavitary bacillus calmette-guerin in the treatment of superficial bladder tumors. J Urol. (1976) 116:180-3. doi: 10.1016/S0022-5347(17)58737-6

23. Potretzke A, Hillman L, Wong K, Shi F, Brower R, Mai S, et al. NLR is predictive of upstaging at the time of radical cystectomy for patients with urothelial carcinoma of the bladder. Urol Oncol. (2014) 32:631-6. doi: 10.1016/j.urolonc.2013.12.009

24. Power NE, Izawa J. Comparison of guidelines on non-muscle invasive bladder cancer (EAU, CUA, AUA, NCCN, NICE). Bladder Cancer (2016) 2:27-36. doi: 10.3233/BLC-150034

25. Spiess PE, Agarwal N, Bangs R, Boorjian SA, Buyyounouski MK, Clark PE, et al. Bladder cancer, version 5.2017, NCCN clinical practice guidelines in oncology. J. Natl. Compr. Canc. Netw. (2017) 15:1240-67. doi: 10.6004/jnccn.20 17.0156

Conflict of Interest Statement: The authors declare that the research was conducted in the absence of any commercial or financial relationships that could be construed as a potential conflict of interest.

Copyright (c) 2019 Yuk, Jeong, Kwak, Kim and Ku. This is an open-access article distributed under the terms of the Creative Commons Attribution License (CC BY). The use, distribution or reproduction in other forums is permitted, provided the original author(s) and the copyright owner(s) are credited and that the original publication in this journal is cited, in accordance with accepted academic practice. No use, distribution or reproduction is permitted which does not comply with these terms. 